\title{
Propiedades de resistencia de una pulpa kraft de pino mezclada con médula del bagazo de caña de azúcar
}

\author{
José Guadalupe Rutiaga Quiñones ${ }^{1}$ \\ José Anzaldo Hernández ${ }^{2}$ \\ José de Jesús Vargas Radillo \\ Rubén Sanjuán Dueñas ${ }^{2}$
}

\begin{abstract}
RESUMEN
Se mezcló pulpa kraft blanqueada de Pinus douglasiana con médula blanqueada de bagazo de caña Saccharum officinarum y se formaron hojas estándar de acuerdo con las normas TAPPI. Para evaluar estadísticamente los efectos de la adición de médula sobre el largo de ruptura y el índice de rasgado en hojas estándar se aplicó un diseño experimental factorial $(3 \times 5)$ (factor $\mathrm{A}=$ grado de refinación de la pulpa: $12^{\circ} \mathrm{SR}, 30^{\circ} \mathrm{SR}$ y $60{ }^{\circ} \mathrm{SR}$; factor $\mathrm{B}=$ cantidad de médula: $0 \%, 10 \%, 20 \%, 30 \%$ y $40 \%$ ). Los resultados indican que la médula de bagazo de caña ayuda a incrementar el largo de ruptura, sobre todo en las pulpas con $12^{\circ} \mathrm{SR}$ y $30^{\circ} \mathrm{SR}$. Se encontraron diferencias estadísticas para ambas variables entre la pulpa con $12^{\circ} \mathrm{SR}$ y las refinadas a $30^{\circ} \mathrm{SR}$ y $60^{\circ} \mathrm{SR}$; mientras que, estadísticamente, los resultados de las pulpas refinadas a $30^{\circ} \mathrm{SR}$ y $60^{\circ} \mathrm{SR}$ fueron similares.

PALABRAS CLAVE:

Índice de rasgado, largo de ruptura, médula, Pinus douglasiana, pulpa kraft, Saccharum officinarum.
\end{abstract}

\begin{abstract}
Bleached kraft pulp of Pinus douglasiana was mixed with bleached pith of sugarcane bagasse and handsheets were made according to TAPPI methods. A factorial design experiment $(3 \times 5)$ was applied for the evaluation of the effects of pith on the breaking length and the tear index of handsheets (factor $A=$ refining degree pulp: 12 ${ }^{\circ} \mathrm{SR}, 30^{\circ} \mathrm{SR}$ and $60{ }^{\circ} \mathrm{SR}$; factor B = quantity of pith: $0 \%, 10 \%, 20 \%, 30 \%$ and $40 \%$ ). The results showed that the pith helped to increase the breaking length, especially in pulp with $12^{\circ} \mathrm{SR}$ and $30^{\circ} \mathrm{SR}$. Statistical differences for both response variables between the pulp with $12^{\circ} \mathrm{SR}$ and the pulp refined to $30^{\circ} \mathrm{SR}$ and $60^{\circ} \mathrm{SR}$ were found, whereas, statistically, the results for the two pulps refined to $30^{\circ} \mathrm{SR}$ and 60 were similar.
\end{abstract}

KEY WORDS:

Tear index, breaking length, pith, Pinus douglasiana, kraft pulp, Saccharum officinarum. 


\section{INTRODUCCIÓN}

La caña de azúcar es una planta tropical y subtropical cultivada en una zona alrededor del Ecuador que llega a los 30 grados de latitud Norte y Sur que fue clasificada por Linneo en 1753 como Saccharum officinarum L. (GEPLACEA, 1990). Esta planta se desarrolla en muchas regiones de la República Mexicana y juega un papel muy importante, tanto en la producción azucarera, como en la producción de pulpa celulósica, una vez extraído el jugo azucarado. En la zafra de 2000/2001 se molieron 44478654 t de caña en los 60 ingenios azucareros que operaron en el país (www.sagarpa.gob.mx). El bagazo de caña de azúcar es una materia prima importante en la fabricación de papel y su empleo inició hace más de 150 años (Zegarra, 1979). La Cámara Nacional de las Industrias de la Celulosa y del Papel, en su memoria estadística de 2001 (CNIDCP, 2001), indica que en el año 2000 fueron producidas 199755 toneladas métricas de celulosa química blanqueada de caña de azúcar, representado una participación relativa del $34,3 \%$. El bagazo de caña de azúcar, cuya composición morfológica es de aproximadamente $50 \%$ de fibras, $30 \%$ de parénquima (médula), 13\% de elementos de vaso y $5 \%$ de epidermis (GEPLACEA, 1990), ha sido motivo de muchos estudios, entre ellos algunos que tratan temas sobre su empleo en la fabricación de papel y de cartón (Zegarra y Zárate, 1977; Atchison, 1979, 1987; Amador y Carrasco, 1979; Giertz, 1979; Betancourt, 1981; Sidney, 1987, 1991; Matta, 1991a, 1991b), otros sobre procesos de pulpeo (Montes, 1973; Sanjuán, 1988; Ramos, 1989). Algunos estudios alrededor de la médula han abarcado temas como el mejoramiento de las propiedades ópticas en pulpas de bagazo de caña (Carreón, 1994), el factor de compresibilidad (Rodríguez, 1996) y las técnicas de blanqueo (Rutiaga et al., 2002). Se considera entonces importante ampliar el conocimiento sobre las carac-terísticas de la médula en cuanto a la influencia en las propiedades de resistencia de pulpas celulósicas a partir de madera, pues en la literatura (Zegarra 1979; ICIDICA, 1986; Matta, 1991a) se menciona que este material afecta dichas propiedades en pulpas de bagazo de caña.

\section{OBJETIVO}

Evaluar estadísticamente el efecto de la combinación de médula blanqueada de bagazo de caña de azúcar sobre dos propiedades de resistencia, largo de ruptura e índice de rasgado, en la pulpa kraft blanqueada de Pinus douglasiana a diferentes grados de refinación.

\section{METODOLOGÍA}

La madera de Pinus douglasiana Martínez fue obtenida del predio denominado Las Tasajeras del Municipio de Mascota, Jalisco, México. Muestras de esta madera fueron pulpeadas mediante el proceso kraft y la pulpa celulósica obtenida fue blanqueada mediante la secuencia $\mathrm{OD}_{1} \mathrm{D}_{2}$ (Oxígeno-Dióxido de cloro-Dióxido de cloro). Información sobre las condiciones del proceso de pulpeo y blanqueo, así como de las características fisicomecánicas de esta pulpa ya se ha presentado en otro trabajo (Rutiaga et al., 1998). El bagazo de caña de azúcar, $S$. officinarum L., fue recolectado de la zafra 1995/1996 en el ingenio azucarero José María Martínez S.A. de Tala, Jalisco, México. El bagazo, seco al aire libre, fue desmedulado en seco en una criba vertical y, posteriormente, la fracción de médula fue tamizada y luego blanqueada mediante la secuencia $\mathrm{OD}_{1} \mathrm{E}_{\mathrm{p}} \mathrm{D}_{2}$ (OxígenoDióxido de cloro-Extracción con PeróxidoDióxido de cloro). Información sobre el proceso de desmedulado, blanqueo y propiedades de la médula blanqueada también ha sido publicada previamente (Rutiaga et al., 2002). Para evaluar estadísticamente el efecto de la mezcla de médula blanqueada con pulpa kraft de $P$. douglasiana blanqueada sobre el largo de ruptura y el índice de rasgado se aplicó un 
diseño experimental tipo factorial (3x5) (Montgomery, 1991), teniendo los siguientes factores y niveles:

$\mathrm{A}=$ grado de refinación ('SchopperRiegler, ${ }^{\circ} \mathrm{SR}$ ) de la pulpa kraft de pino (12 ${ }^{\circ} \mathrm{SR}, 30^{\circ} \mathrm{SR}$ y $\left.60{ }^{\circ} \mathrm{SR}\right)$

$\mathrm{B}=$ volumen de adición de médula $(0 \%$, $10 \%, 20 \%, 30 \%, 40 \%)$, con dos repeticiones

Aquí es necesario hacer la siguiente observación: a la pulpa sin refinar, a pesar de no haber sido sometida a ese tratamiento mecánico de refinación, se le midió el grado de refinación resultando $12{ }^{\circ} \mathrm{SR}$; de manera que, al hablar de pulpa sin refinar, se refiere precisamente a la pulpa con el valor en el grado de refinación de $12{ }^{\circ} \mathrm{SR}$.

El modelo estadístico correspondiente a tal diseño es (Montgomery, 1991):

$$
y_{i j k}=\mu+\tau_{i}+\beta_{j}+(\tau \beta)_{i j}+\varepsilon_{i j k}
$$

donde:

$$
\begin{aligned}
y_{i j k}= & \text { variable de respuesta } \\
\mu & =\text { media general o efecto común } \\
\tau_{i} & =\text { efecto del grado de refinación } \\
\beta_{j} \quad= & \text { efecto debido a la adición de } \\
& \text { médula } \\
(\tau \beta)_{i j}= & \text { efecto de la interacción de los } \\
& \text { factores } \mathrm{AB} \\
\varepsilon_{i j k}= & \text { error aleatorio }
\end{aligned}
$$

Las variables de respuesta fueron el largo de ruptura y el índice de rasgado. Como ejemplo de las hipótesis estadísticas que se pueden probar con dicho modelo se ilustran sólo para el largo de ruptura:

$$
\begin{aligned}
\mathrm{H}_{0}= & \text { no hay efecto del grado de refinación } \\
& \text { sobre el largo de ruptura } \\
\mathrm{H}_{1}= & \text { sí hay efecto } \\
\mathrm{H}_{0}= & \text { no hay efecto de la adición de } \\
& \text { médula sobre el largo de ruptura } \\
\mathrm{H}_{1}= & \text { sí hay efecto }
\end{aligned}
$$

$H_{o}=$ no hay efecto de la interacción entre estos dos factores

$\mathrm{H}_{\mathrm{o}}=$ sí hay efecto

El nivel de confianza estadística con que se analizaron los datos fue del $95 \%$, evaluando los resultados mediante el programa Statgraphics versión 6.0 (Gutiérrez et al., 1996). Para hacer las comparaciones de las medias de los efectos significativos se aplicó la prueba de la mínima diferencia significativa LSD (Montgomery, 1991). Para mostrar las tendencias del efecto de adicionar médula a la pulpa celulósica sobre el largo de ruptura e índice de rasgado, se utilizaron los modelos matemáticos resultantes del tratamiento de los datos mediante el programa Origin versión 2.94. La pulpa blanqueada de pino fue refinada a $30{ }^{\circ} \mathrm{SR}$ y $60^{\circ} \mathrm{SR}$ en un molino Jokro. Las hojas estándar de $60 \mathrm{~g} / \mathrm{m}^{2}$ (hojas de 1,2 g) fueron formadas con las mezclas de médula y las pulpas (con $12{ }^{\circ} \mathrm{SR}, 30^{\circ} \mathrm{SR}$ y $60{ }^{\circ} \mathrm{SR}$ ) en un equipo convencional TAPPI semiautomático. Las normas para la preparación y evaluación de las propiedades de resistencia de las hojas estándar aparecen en la Tabla 1.

\section{RESULTADOS Y DISCUSIÓN}

Las figuras 1 y 5 muestran las tendencias sobre el largo de ruptura y el índice de rasgado al adicionar médula a la pulpa de pino, respectivamente; se presentan también las ecuaciones matemáticas, con buenas correlaciones, aplicables a las condiciones de esta experimentación.

Ecuaciones para el largo de ruptura:

$$
\begin{aligned}
& y_{12}{ }^{\circ} S R=1282,06+318,75 x-4,96 x^{2} \\
& r \quad=0,96 \\
& r^{2}=0,93 \\
& D E=819,85 \mathrm{~m}
\end{aligned}
$$




$$
\begin{aligned}
& y_{30}{ }^{\circ} \mathrm{SR}=6063,88+73,18 x-1,29 x^{2} \\
& r \quad=0,99 \\
& r^{2}=0,98 \\
& D E=79,26 \mathrm{~m} \\
& y_{60}{ }^{\circ} \mathrm{SR}=7038,9+74,97 x-2,14 x^{2} \\
& r \quad=0,95 \\
& r^{2}=0,89 \\
& D E=211,06 \mathrm{~m}
\end{aligned}
$$

En la figura 1 se observa el efecto que produce el adicionar médula a la pulpa kraft sobre el largo de ruptura. Se ve claramente que hay un incremento en el valor de la variable de respuesta, sobre todo en la pulpa sin refinar $y$, en casi todos los casos, es mayor que el control, es decir sin médula. Para el caso de la pulpa con $12{ }^{\circ} \mathrm{SR}$, estos incrementos fueron del $517 \%$, 619\%, 621\% y $708 \%$, al adicionar $10 \%, 20 \%, 30 \%$ y $40 \%$ de médula, respectivamente. En este caso, si se requiriera un alto valor del largo de ruptura la cantidad óptima de médula pudiera ser hasta del $40 \%$. Para el caso de la pulpa refinada a $30{ }^{\circ} \mathrm{SR}$ al adicionar $10 \%, 20 \%, 30 \%$ y $40 \%$ de médula, los incrementos fueron del 108\%, $116 \%$, $115 \%$ y $114 \%$, respectivamente; a partir de un $40 \%$ de médula la tendencia es a

Tabla 1. Normas aplicadas para la evaluación de las hojas ( $T=$ TAPPI)

\begin{tabular}{ll}
\hline CLAVE & NOMBRE \\
\hline T 205 om-88 & Formación de hojas \\
T 402 om-93 & Acondicionamiento de las hojas \\
T 404 cm-92/ISO 1924 & Largo de ruptura \\
T 410 om-93 & Gramaje \\
T 411 om-89 & Espesor \\
T 412 om-94 & Por ciento de humedad \\
T 414 om-88/ISO 1974 & Índice de rasgado \\
\hline
\end{tabular}

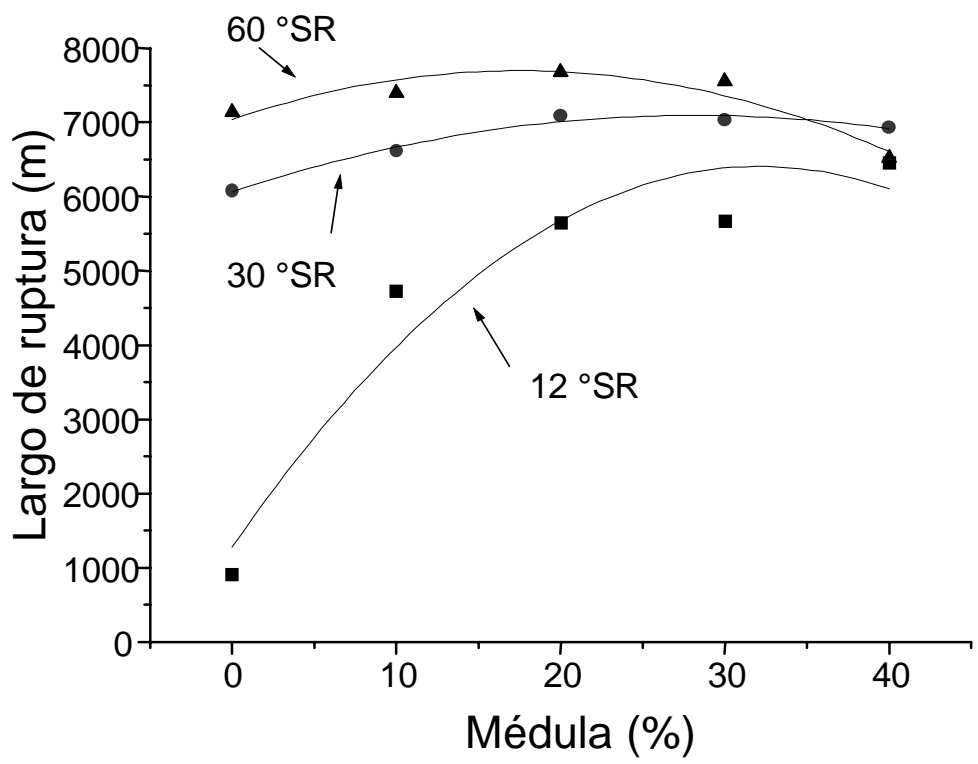

Figura 1. Efecto de la adición de médula a la pulpa kraft de pino sobre el largo de ruptura. 
disminuir, así que el valor óptimo de adición para este caso pudiera ser un $20 \%$. Para la pulpa a $60{ }^{\circ} \mathrm{SR}$ el incremento que se logra al adicionar médula parece estacionarse entre un 10\% y un $30 \%$, bajando a partir del $40 \%$ de adición; con $10 \%, 20 \%$ y $30 \%$ de médula el largo de ruptura se incrementa en $103 \%, 107 \%$ y $105 \%$, respectivamente; con un $40 \%$ de adición ya hay una pérdida del $9 \%$; el valor óptimo en este caso pudiera ser $20 \%$.

Del análisis de varianza para el largo de ruptura se obtuvo un nivel de significancia para el efecto del grado de refinación de 0,0001, para el porcentaje de adición de médula de 0,0016 y para la interacción del grado de refinación y la cantidad de médula de 0,0019, valores que son menores al nivel de significancia fijado de 0,05, por lo tanto, las hipótesis planteadas se rechazan. Esto significa que el grado de refinación, la adición de médula y la interacción entre estos dos factores sí tienen un efecto estadísticamente significativo sobre el largo de ruptura (Fig. 2, 3 y 4).

Como las hipótesis se han rechazado, la prueba de rangos múltiples indica que, estadísticamente, las pulpas a $30{ }^{\circ} \mathrm{SR}$ y 60 'SR son iguales; entonces, si se tomara en cuenta el costo del proceso de refinación será mejor refinar la pulpa a $30{ }^{\circ} \mathrm{SR}$ que a $60{ }^{\circ} \mathrm{SR}$, obteniendo el mismo resultado estadísticamente (Fig. 1, 2 y 4). Por otro lado, esta misma prueba indica que las adiciones de médula de $10 \%$, $20 \%$ y $30 \%$ son estadísticamente iguales y éstas son distintas comparadas con $0 \%$ y $40 \%$ de adición de médula, de modo que un valor óptimo puede ser $20 \%$ de médula (Fig. 3 y 4 ).

Una hoja fabricada con pulpa sin refinar no tiene mucha resistencia (Robinson, 1991), entonces, una explicación de que el uso de la médula ayude a incrementar el largo de ruptura, sobre todo en pulpa sin refinar puede ser porque

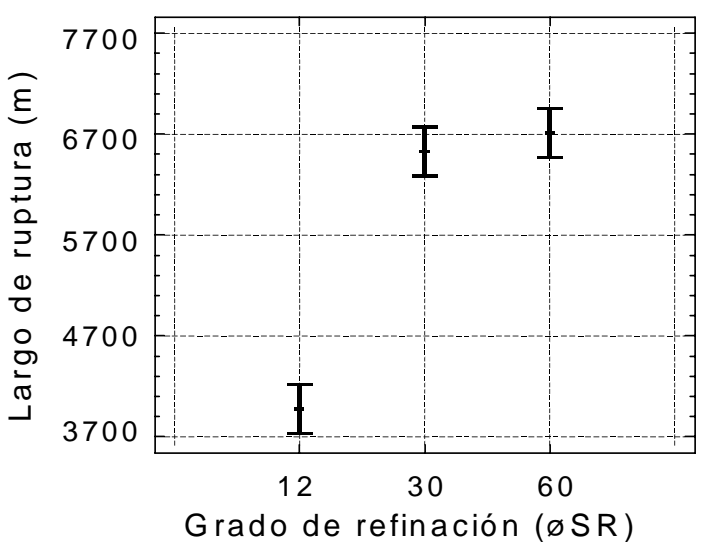

Figura 2. Efecto de la refinación sobre el largo de ruptura.

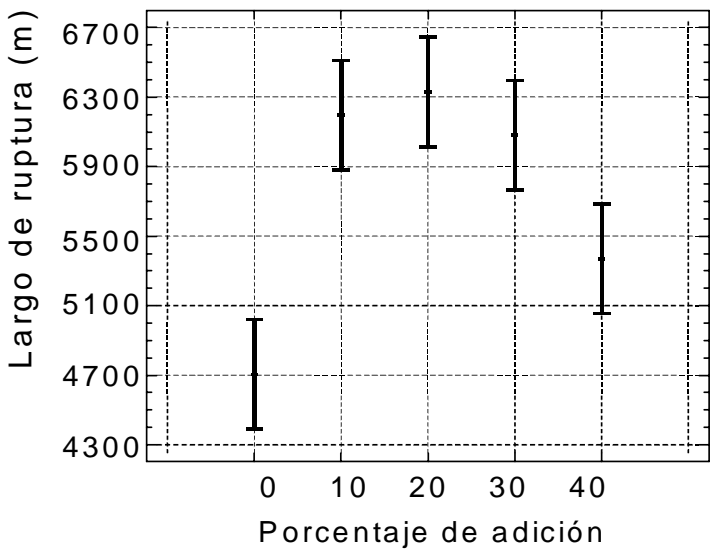

Figura 3. Efecto del porcentaje de adición de médula sobre el largo de ruptura.

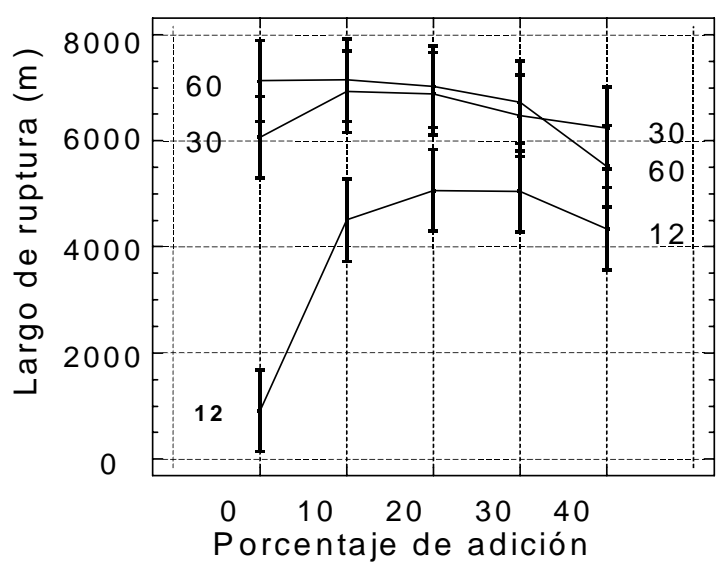

Figura 4. Interacción grado de refinaciónporcentaje de adición. 
es compatible con las fibras, pudiendo así favorecer el desarrollo de enlaces entre estos materiales; además, es de suponerse que la médula actúa como una "malla" o como "finos" que se entrelazan con las fibras, aumentando así el área de contacto, es decir, la superficie dispuesta para la unión y si esto sucede, como lo mencionan Swanson y Steber (1959) y Young (1991), la resistencia a la tensión se incrementa. Algunos autores (Thomson et al., 1953; Clark, 1969) han encontrado que las hemicelulosas actúan como adhesivos ayudando a que los papeles tengan mayor resistencia, ya que el enlace entre fibras se mejora porque aumenta el contacto entre las superficies que contienen grupos hidroxilo. Entonces, de acuerdo con los resultados obtenidos en el presente trabajo (Fig. 1), pudiera considerarse a la médula como un material adhesivo, toda vez que se ha encontrado que tiene un alto contenido de polisacáridos, sobre todo de xilosa, 21,9\% (Camacho, 1983). Al refinar una pulpa se incrementa el área de contacto (Swanson y Steber, 1959) o sea la superficie que participa en la unión (Young, 1991), aumentando también la flexibilidad y plasticidad de las fibras (Robinson, 1991), favoreciendo así un mayor número de enlaces físicos.
Con la adición de médula se promueve el mecanismo esencial mediante el cual las superficies de estos dos materiales pueden lograr un mayor contacto que precede a la formación de enlaces, ayudando así a que el largo de ruptura se incremente (Fig. 1). Para el caso en que se mezcla médula con pulpa muy refinada (60 ${ }^{\circ} \mathrm{SR}$ ) no hay mucha ganancia en el largo de ruptura, ya que la propia fibra ha sido cortada como consecuencia del proceso de refinación y, como mencionan Van Den Akker et al. (1958), una pulpa muy refinada tiene poca resistencia (Fig. 1); además de que la médula, a pesar de no haber sido refinada, ya de por sí presenta un alto "grado de refinación", 42 `SR (Rutiaga et al., 2002).

La figura 5 muestra el efecto de mezclar pulpa kraft con médula sobre el índice de rasgado. Para el caso de la pulpa con $12{ }^{\circ} \mathrm{SR}$, al agregar un $10 \%$ de médula este índice se incrementa en 1,15 veces el valor inicial y con $20 \%$ el índice es similar al valor original, pero si agrega un $30 \%$ y $40 \%$ hay pérdida, por lo que el valor óptimo de adición es de 10\%. Para el caso de la pulpa a $30^{\circ} \mathrm{SR}$ no se tiene una cantidad óptima de médula, ya que en todos los casos no hay ganancia en el índice de rasgado y en la pulpa refinada a

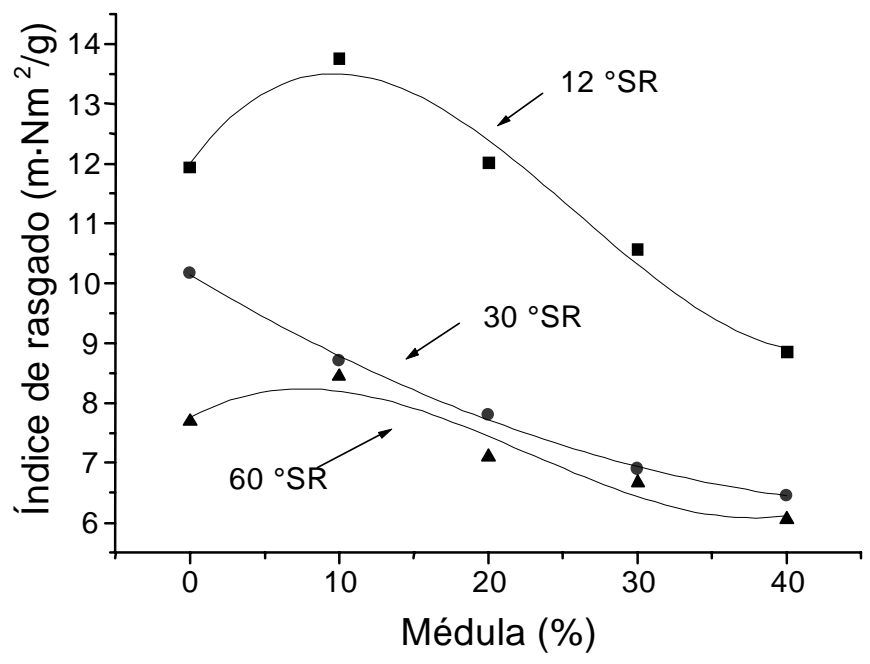

Figura 5. Efecto de la adición de médula a la pulpa kraft de pino sobre el índice de rasgado. 
60 `SR sólo un $10 \%$ de médula muestra un incremento en el índice de rasgado. rasgado:

Ecuaciones para el índice de

$$
\begin{aligned}
& y_{12^{\circ} S R}=12,01+0,33 x-0,02 x^{2}+2,73 \times 10^{4} x^{3} \\
& r \quad=0,99 \\
& r^{2}=0,98 \\
& D E=0,53 \mathrm{~m} \cdot \mathrm{Nm}^{2} / \mathrm{g}
\end{aligned}
$$

$$
y_{30}{ }^{\circ} S R=10,14-0,15 x+0,001 x^{2}
$$$$
r_{2}=0,99
$$$$
r^{2}=0,99
$$$$
\mathrm{DE}=0,09 \mathrm{~m} \cdot \mathrm{Nm}^{2} / \mathrm{g}
$$$$
y_{60}{ }^{\circ} S R=7.76+0,14 x-0,01 x^{2}+1,57 \times 10^{-4} x^{3}
$$$$
\begin{array}{ll}
r & =0,96 \\
r^{2} & =0,93 \\
D E & =0,48 \mathrm{~m} \cdot \mathrm{Nm}^{2} / \mathrm{g}
\end{array}
$$

El análisis de varianza realizado para el índice de rasgado indicó los siguientes valores de nivel de significancia: 0,0001 para el grado de refinación, 0,0001 para el factor de adición de médula y 0,0006 para la interacción del grado de refinación y el porcentaje de médula. Debido a que estos valores calculados son menores al nivel de significancia fijado de 0,05, las hipótesis probadas se rechazan, ya que estos factores sí tienen efecto estadístico sobre el índice de rasgado (Fig. 6, 7 y 8).

Al rechazar las hipótesis, la prueba de rangos múltiples indica que para el grado de refinación, las pulpas con $30^{\circ} \mathrm{SR}$ y $60{ }^{\circ} \mathrm{SR}$ son estadísticamente iguales y comparadas éstas con la pulpa a $12{ }^{\circ} \mathrm{SR}$ son estadísticamente diferentes (Fig. 6). Como en la industria la pulpa debe ser refinada, ésta deberá refinarse a $30^{\circ} \mathrm{SR}$ puesto que se requerirá menor energía que si se refinara a $60{ }^{\circ} \mathrm{SR}$; además con $30{ }^{\circ} \mathrm{SR}$ el índice de rasgado se afectaría menos (Fig. 6). Por otra parte, para el porcentaje de médula, la prueba de rangos múltiples indica que los porcentajes de adición de médula son

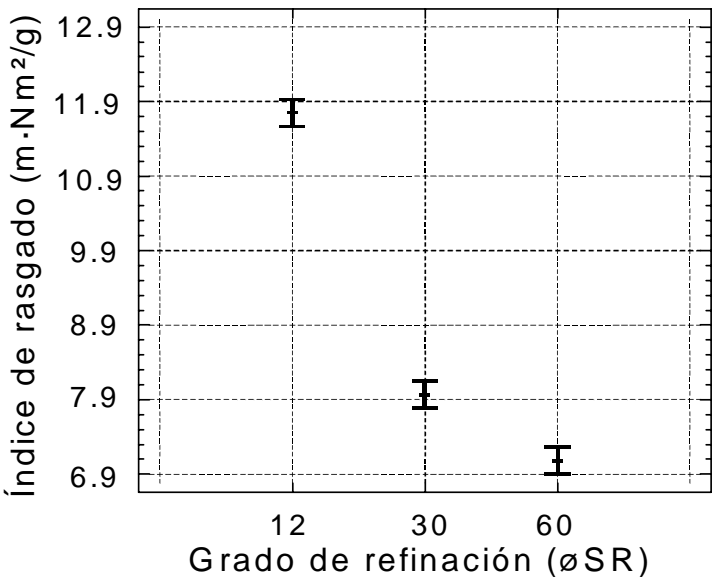

Figura 6. Efecto del grado de refinación sobre el índice de rasgado.

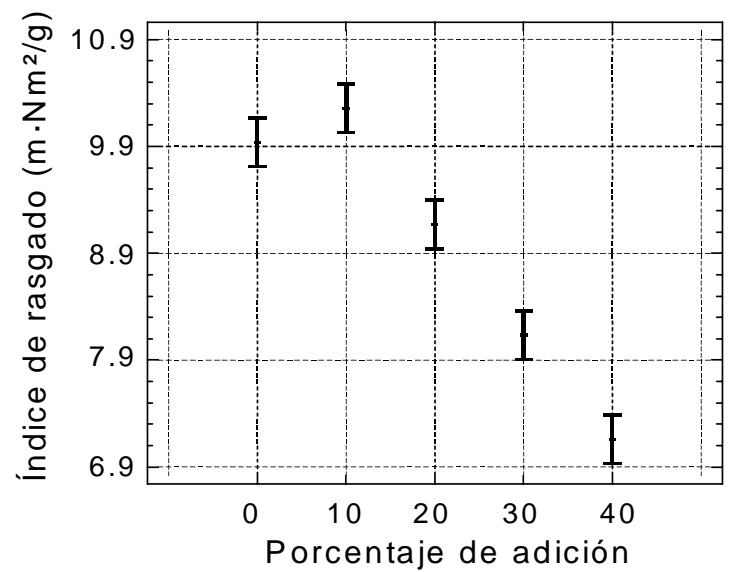

Figura 7. Efecto del porcentaje de adición de médula sobre el índice de rasgado.

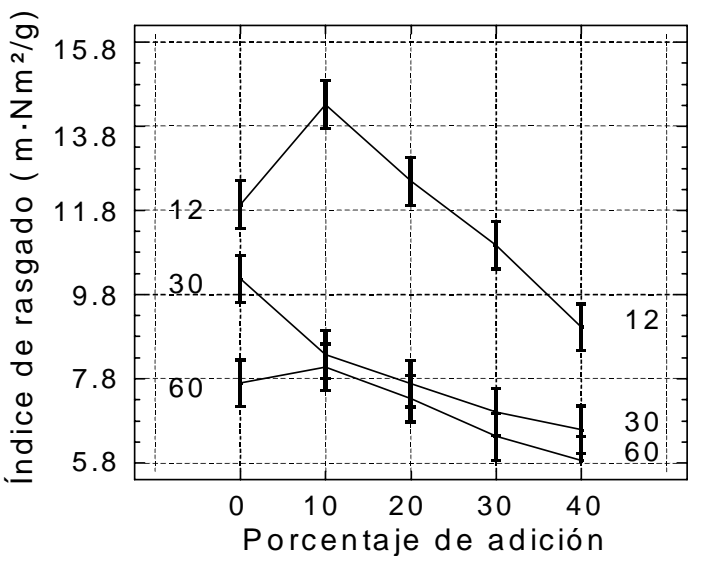

Figura 8. Interacción grado de refinaciónporcentaje de adición. 
estadísticamente diferentes entre sí, en este caso un valor óptimo de médula puede ser de 10\% (Fig. 7 y 8 ).

Como la médula promueve el desarrollo de enlaces entre sí y las fibras, hay una concentración de la resistencia al rasgado en una zona pequeña, disminuyendo la propensión al rasgado, tal como lo menciona Brandon (1991). De manera semejante, la resistencia al rasgado de una hoja se determina casi totalmente por el largo de las fibras que la forman (Clark, 1969), de modo que si en la prueba participan menos fibras largas de la pulpa de pino y más material parenquimatoso (médula) la resistencia al rasgado disminuirá (Fig. 5, 7 y 8 ).

\section{CONCLUSIONES}

Se encontró que la adición de médula ayuda a incrementar el largo de ruptura de la pulpa kraft blanqueada de pino, sobre todo en las pulpas con $12^{\circ} \mathrm{SR}$ y 30 'SR; el valor óptimo de adición de médula puede ser de $20 \%$. Para el caso del índice de rasgado, se encontró que una adición de sólo $10 \%$ de médula ayuda a incrementarlo en la pulpa kraft con 12 `SR. Los demás porcentajes causan una pérdida en esta variable de respuesta en las tres pulpas celulósicas. Para ambas propiedades de resistencia se encontró que las pulpas refinadas a $30{ }^{\circ} \mathrm{SR}$ y $60{ }^{\circ} \mathrm{SR}$ son estadísticamente iguales y que la pulpa con $12{ }^{\circ} \mathrm{SR}$ es estadísticamente diferente comparada con las pulpas refinadas a $30^{\circ} \mathrm{SR}$ y $60{ }^{\circ} \mathrm{SR}$. Se encontró que es factible emplear hasta $20 \%$ de médula blanqueada para incrementar el largo de ruptura de la pulpa kraft blanqueada de pino.

\section{REFERENCIAS}

Amador, J. y S. Carrasco. 1979. The bagasse pulp and paper industry in Mexico. Non-wood Plant Fiber Pulping. Tappi Report 10:1-22.
Atchison, J.E. 1979. Historical development of bagasse newsprint processes prosed and mills built, present status of bagasse newsprint technology, and possibilities for the future. Non-wood Plant Fiber Pulping. Tappi Report 10:71-90.

Atchison, J.E. 1987. Critical factors to be considered in production of bagasse newsprint and the necesary high yield mechanical pulp required. Nonwood Plant Fiber Pulping. Tappi report 17:63-101.

Betancourt T., J. 1981. Prespectivas de aprovechamiento del bagazo de caña en la industria de la celulosa y papel. ATCP XXI(4):213-221.

Brandon, C.E. 1991. Propiedades del papel. In: J.P. Casey, ed. Pulpa y papel, química y tecnología química. LIMUSA. México. Vol III. cap. 21. pp 321-601.

Camacho L., A. 1983. Estudio de la transformación por fermentación de la médula de bagazo de caña en piensos. Tesis de maestría. Universidad de Guadalajara. México. 69 p.

Cámara Nacional de las Industrias de la Celulosa y del Papel. Memoria Estadística 2001. México. 86 p.

Carreón R., H.D. 1994. Mejoramiento de las calidades ópticas de la pulpa de bagazo de caña. Tesis de Licenciatura. Universidad de Guadalajara. México. $77 \mathrm{p}$.

Clark, J.d'A. 1969. Fibrillation, free water, and fiber bonding. Tappi 52(2):335340.

GEPLACEA, CUBA-9, PNUD, ICIDICA. 1990. Atlas del bagazo de caña de azúcar. México. 143 p.

Giertz, H.W. 1979. Aspects on the problem of producing newsprint from 
bagasse. Non-wood Plant Fiber Pulping. Tappi Report 10:91-98.

Gutiérrez P., H.; P. Gutiérrez G. y O. Camacho C. 1996. Análisis estadístico por computadora - Statgraphics. Ediciones Aleph. México. 189 p.

ICIDICA. 1986. La industria de los derivados de la caña de azúcar. Editorial Científico-Técnica. La Habana. 576 p.

Matta, J.E. 1991a. Papel periódico con bagazo y/o caña. I. Fundamentos de su comportamiento. ATIPCA 30(1): 45-55.

Matta, J.E. 1991b. Papel periódico con bagazo y/o caña. II. Potencionalidad y manejo de las fibras. ATIPCA 30(1):56-62.

Montes R., E. 1973. Obtención de pulpas celulósicas de diferentes calidades a partir de bagazo de caña. Tesis de Licenciatura. Universidad de Guadalajara. México. $51 \mathrm{p}$.

Montgomery, D.C. 1991. Diseño y análisis de experimentos. Grupo Editorial Iberoamérica. México. 589 p.

Ramos Q., J. 1989. Producción de pulpas quimicomecánicas con varios reactivos químicos a partir de bagazo de caña. Tesis Doctoral. Universidad de Guadalajara. México. 468 p.

Rodriguez B., V.M. 1996. Determinación del factor de compresibilidad de la médula de bagazo y su influencia en las propiedades físico mecánicas del papel. Tesis de Licenciatura. Universidad de Guadalajara. México. 109 p.

Rutiaga Q., J.G., J.J. Rivera P. y R. Sanjuán D. 1998. Evaluación fisicomecánica de la pulpa Kraft blanqueada de la madera de Pinus douglasiana Martínez. Ciencia Forestal en México. 23(83):17-31.
Rutiaga Q., J.G., J.J. Rivera P. y R. Sanjuán D. 2002. Estudio sobre el parénquima de Saccharum officinarum L. Enviado, Revista Ciencia Nicolaita.

Robinson, J.V. 1991. Unión de las fibras. In: J.P. Casey, ed. Pulpa y papel química y tecnología química. Ed. LIMUSA. México. Vol II. cap. 7. pp:137-195.

Sanjuán D., R. 1988. Investigaciones en procesos de pulpeo con aplicación de etanol utilizando bagazo de caña. Tesis Doctoral. Universidad de Guadalajara. México. 208 p.

Sidney, G.E. 1987. TMP and CTMP bagasse pulp experienced from Mexican fiberboard mill. Non-wood Plant Fiber Pulping. Tappi Report 17:53-57.

Sidney, G.E. 1991. Mexican mills utilizing bagasse to produce pulp and fiberboard mill. Non-wood Plant Fiber Pulping. Tappi Report 19:45-70.

Swanson, J.W. y A.J. Steber. 1959. Fiber surface area and bonded area. Tappi 42(12):986-994.

TAPPI Test Methods (1994-1995). 1994. TAPPI Press. Atlanta. EUA.

Thomson, J.O.; J.W. Swanson y L.E. Wiese. 1953. Hemicelluloses and arabogalactans as beater adhesives. Tappi 36(12):534-541.

Van Den Akker, J.A.; A.L. Lathrop; M.H. Voelker y L.R. Dearth. 1958. Importance of fiber strength to sheet strength. Tappi 41(8):416-425.

www.sagarpa.gob.mx/coaazucar/menu5/s emanal4.htm

Young, J.H. 1991. Preparación de la fibra y flujo de alimentación de pasta. In: J.P. Casey, ed. Pulpa y papel química y tecnología química. Ed. 
LIMUSA. México. Vol II. cap. 6. Zegarra, J.R. y J. Zárate. 1977. Paper pp:25-136. from bagasse. Non-wood Plant Fiber Pulping. Tappi Report 8:3-8. $\diamond$

\section{Zegarra, J.R. 1979. Consideraciones sobre el desmedulado del bagazo. ATCP XIX(2):95-102.}

$1 \quad$ Facultad de Ingeniería en Tecnología de la Madera, Universidad Michoacana de San Nicolás de Hidalgo. Fco. J. Mújica s/n. Edificio “D” planta alta. C. U. Morelia 58041 Michoacán, México. rutiaga@ zeus.umich.mx.

2 Departamento de Madera, Celulosa y Papel Ing. Karl Augustin Grellmann. Universidad de Guadalajara. Apdo. Postal 52-93. Zapopan 45020, Jalisco, México. janzaldo@dmcyp.cucei.udg.mx, jvradillo@ hotmail.com, rsanjuan@dmcyp.cucei.udg.mx.

Manuscrito recibido el 14 de septiembre de 2000.

Aceptado el 6 de febrero de 2001.

Este documento se debe citar como:

Rutiaga Q., J.G.; J. Anzaldo H.; J.J. Vargas R. y R. Sanjuán D. 2002. Propiedades de resistencia de una pulpa kraft de pino mezclada con médula de bagazo de caña de azúcar. Madera y Bosques 8(2):17-26. 